\title{
Role of the preprobe delay in memory-scanning tasks
}

\author{
DON DIENER \\ University of Nevada, Las Vegas, Nevada
}

\begin{abstract}
In a variation of Sternberg's $(1966,1969)$ memory-scanning task not requiring an explicit negative response, Diener (1988) found that a preprobe delay was necessary to produce the usual setsize effect. In Experiment 1 of the present study, the effect of the preprobe delay was investigated in the typical two-response task. In the absence of a preprobe delay, the function relating response latency to set size was virtually flat for negative responses, but was described by a slope of about $18 \mathrm{msec} /$ item for positive responses. Further research suggested that the reduced set-size effect in the absence of a preprobe delay is the result of expectancy effects usually controlled by the preprobe delay. Informing the subject of the size of the memory set before it was presented (Experiment 2) produced a set-size effect of the usual magnitude in the absence of a preprobe delay. Experiment 3 was designed to assess the effects of expectancy in the absence of a memory search. A task similar in stimulus arrangement to the memory-scanning task but requiring the subject to indicate whether the last digit in the set was odd or even produced a decrease in response latency with set size of $29 \mathrm{msec} / \mathrm{item}$ in the absence of a preprobe delay.
\end{abstract}

Diener (1988, Experiment 3) presented subjects with series of two to seven digits, followed immediately by a test digit, identified only by its print style. Subjects were instructed to press a key if the test digit had appeared in the series. If the test digit had not been previously displayed, the subjects were instructed not to respond. This procedure differed from the widely studied memoryscanning task developed by Sternberg $(1966,1969)$ in that (1) no explicit negative response was required and (2) the test digit followed the memory set immediately with no preprobe delay and no warning that the next digit to be presented would serve as a probe.

In Sternberg's task, response latency typically increases linearly with set size at the rate of about $35 \mathrm{msec} / \mathrm{item}$. The above-described procedure resulted in a set-size effect of only about $9 \mathrm{msec} / \mathrm{item}$. A set-size effect of the typical magnitude was restored by the introduction of a preprobe delay (Diener, 1988, Experiment 4). A 1-sec delay prior to the presentation of the test digit resulted in a reduction in mean response latency of about $140 \mathrm{msec}$ and a set-size effect of $31 \mathrm{msec} /$ item. These results suggest that a preprobe delay is necessary for the occurrence of the typical set-size effect, at least in tasks that do not require an explicit negative response.

The absence of a preprobe delay has not been studied in a task requiring an explicit negative response. There are several reasons for doing so. First, the requirement of an explicit negative response may interact with other factors in unexpected ways. Egeth, Marcus, and Bevan (1972) found no effect of increasing the size of the

I wish to thank Kim Foster for her assistance in collecting the data. Requests for reprints should be addressed to Don Diener, Department of Psychology, University of Nevada, Las Vegas, 4505 Maryland Parkway, Las Vegas, NV 89154. memory set from one to three digits when the larger set consisted of a "familiar" sequence $(1,2,3)$ and no explicit negative response was required. When an explicit negative response was required, the usual set-size effect was found for both positive and negative responses regardless of the nature of the memory set. Second, even if requiring an explicit negative response does not alter the basic results, positive and negative responses may be affected by the absence of a preprobe delay in somewhat different ways.

Experiment 1 was conducted to investigate the effects of the preprobe delay on a task requiring an explicit negative response. Performance on a typical two-response memory-scanning task with a 1-sec preprobe delay was compared with performance on a similar task with no preprobe delay

\section{EXPERIMENT 1}

\section{Method}

Subjects. The subjects were recruited from undergraduate psychology classes. Eight men and 12 women, ranging in age from 19 to 49 years (median age $=22.5$ years), participated in the delay condition; 9 men and 11 women, ranging in age from 18 to 48 years (median age $=22.5$ years), participated in the no-delay condition. Three additional subjects participated in the no-delay condition but were replaced because of very high error rates $(45 \%$ to $47 \%$ )

Apparatus. An Apple Ile microcomputer was used to present stimuli, record responses, and measure response latencies. Stimuli were presented on the Apple Monitor II video monitor in the 40 column display format. In this display, numerals and letters are formed on $5 \times 7$ pixel grids (approximately $4 \mathrm{~mm} \times 6 \mathrm{~mm}$ ). The subjects were allowed to observe the display at the distance they found most comfortable.

Procedure. On each trial, a series of one to six digits (0-9) was presented sequentially in the center of the screen at the rate of one 
digit per second. In the no-delay condition, a test digit was displayed immediately after the presentation of the last digit in the series. This test digit was displayed in an inverse format. That is, the numerals were formed by the dark areas of the otherwise illuminated $5 \times 7$ pixel grid. In the delay condition, presentation of the inverse test digit was preceded by a $1-\mathrm{sec}$ interval, during which an illuminated square (an "inverse space") was displayed at the same position the digits appeared. The subjects were instructed to press the $\mathrm{K}$ key on the computer keyboard with the index finger of the right hand if the test digit had appeared in the preceding series, and to press the D key with the index finger of the left hand if the test digit had not been displayed previously. The test digit was displayed until the subject responded.

If the subject's response was incorrect, occurred before the test digit had been displayed for $20 \mathrm{msec}$, or occurred after the test digit had been displayed for $1,500 \mathrm{msec}$, the subject was so informed by an appropriate message briefly displayed on the screen. In the case of any of these errors, the data were excluded from the analysis and the trial was immediately replaced by another with the same series length. Shortly after a response, the subject was instructed by a message on the screen to press the space bar to begin a new trial.

The length of each series and the serial position of the test digit on positive trials were determined by the value of a random number produced by the computer's random function. The system was programmed such that on any trial the probabilities of all series lengths and serial positions were equal. Although all series lengths were equally probable on any given trial, the total number of trials at each series length was not set and varied slightly from subject to subject. As each subject received 160 trials on the task, the expected number of observations per cell was 13.3. The lowest number of trials recorded in any of the 480 cells ( 6 set sizes $\times 2$ response types $\times 40$ subjects) was 6 and the highest was 24 .

A message on the computer screen informed the subjects when half of the trials had been completed and instructed them to take a short break if they so desired. The entire procedure took about $30 \mathrm{~min}$.

\section{Results}

In the delay condition, response latency increased with set size for both positive and negative responses (see Figure 1). In the no-delay condition, response latency increased at a lower rate than it did in the delay condition for positive responses, and did not change appreciably with set size for negative responses.

A mixed (delay $\times$ set size $\times$ response type $\times$ subject within-delay) analysis of variance (ANOVA) performed on the response-latency data confirmed most of the trends apparent in Figure 1. Response latency was significantly longer in the no-delay condition than it was in the delay condition [789 vs. $681 \mathrm{msec} ; F(1,38)=9.42, p<.01]$. The main effect of response type was significant $[F(1,38)$ $=56.19, p<.001]$ due to the longer latencies of negative responses ( 761 vs. $646 \mathrm{msec}$ ). There was no response type $\times$ delay interaction $[F(1,38)=2.49, p>.05]$. The set-size effect was significant $[F(5,190)=33.83$, $p<.001]$. A significant set size $\times$ delay interaction $[F(5,190)=11.41, p<.001]$ confirmed the difference in the slopes between the two conditions. A significant response type $\times$ set size interaction $[F(5,190)=5.56$, $p<.001]$ indicated differences in the slopes of positiveand negative-response functions.

Separate subject $\times$ set size $\times$ response type ANOVAs were performed on the response-latency data from the two

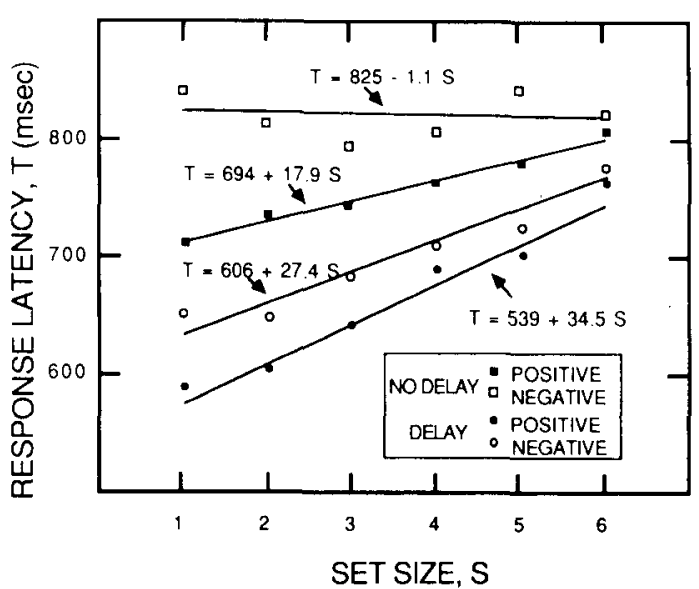

Figure 1. Mean response latency as a function of set size for positive and negative responses in the delay and no-delay conditions of Experiment 1.

conditions. Significant set-size effects were found in both conditions [delay condition, $F(5,95)=31.27, p<.001$; no-delay condition, $F(5,95)=6.20, p<.001]$. The response-type main effects were also significant [delay condition, $F(1,19)=21.14, p<.001$; no-delay condition, $F(1,19)=35.13, p<.001]$. The response type $\times$ set size interaction was significant in the no-delay condition $[F(5,95)=6.47, p<.001]$ but not in the delay condition $[F(5,95)<1]$.

In the delay condition, the regression of response latency on set size for positive responses yielded a slope of $34.46 \mathrm{msec} /$ item $(r=.99)$ and a zero intercept of $539 \mathrm{msec}$. The $95 \%$ confidence interval for the slope, derived from the linear component of a subject $\times$ set size trend analysis, was $\pm 6.62 \mathrm{msec} /$ item. For negative responses, the slope of the response-latency function was $27.36 \mathrm{msec} /$ item $( \pm 6.19 ; r=.98)$ and the intercept was $606 \mathrm{msec}$. In the no-delay condition, the slope of the response-latency function was $17.93 \mathrm{msec} / \mathrm{item}$ for positive responses $( \pm 5.39 ; r=.98)$ and the intercept was $694 \mathrm{msec}$; for negative responses, the slope was $-1.11 \mathrm{msec} /$ item $( \pm 4.97 ; r=-.11)$ and the intercept was $825 \mathrm{msec}$.

The effects of the serial position of the test digit in the memory set on positive trials were analyzed in separate subject $\times$ serial position ANOVAs for each condition and set size. In the delay condition, there was a significant effect of serial position on response latency only for a set size of four digits $[F(3,57)=3.10, p<.05]$. A trend analysis for this set size failed to reveal significant linear, quadratic, or cubic trends, although the quadratic component approached significance $[t(57)=-1.83, p=.07]$, suggesting both primacy and recency effects. In the nodelay condition, significant serial-position effects were found for set sizes of three digits $[F(2,38)=3.34$, $p<.05]$ and four digits $[F(3,57)=3.74, p<.05]$. For the set size of four digits, only the quadratic component of a trend analysis approached significance $[t(57)=$ $-1.97, p=.054]$. Similarly, for the set size of three 
Table 1

Error Rates on Positive and Negative Trials in the Delay and No-Delay Conditions of Experiment 1 as a Function of Set Size

\begin{tabular}{lccccccccc}
\hline & \multicolumn{8}{c}{ Set Size } & \\
\cline { 2 - 7 } & 1 & 2 & 3 & 4 & 5 & 6 & Slope & $r$ \\
\hline Positive & .031 & .058 & .074 & .064 & .099 & .175 & $+.024^{*}$ & .89 \\
Negative & .069 & .055 & .027 & .068 & .100 & .098 & $+.010^{+}$ & .63 \\
& \multicolumn{7}{c}{ No-delay Condition } \\
Positive & .099 & .115 & .094 & .130 & .201 & .170 & $+.018^{*}$ & .82 \\
Negative & .091 & .086 & .062 & .067 & .113 & .119 & +.006 & .52
\end{tabular}

*Set-size effect significant, $p<.01$. + Set-size effect significant, $p<.05$.

digits, only the quadratic component was significant $[t(38)$ $=-2.08, p<.05]$, indicating small primacy and recency effects.

Error rates (see Table 1) were fairly high, even for the unpracticed subjects, in the delay condition (averaging $7.6 \%)$ and quite high in the no-delay condition (11.2\%). Errors increased with set size on both positive and negative trials in both conditions, although the increase was not statistically significant for negative trials in the nodelay condition. There was no evidence of a speedaccuracy tradeoff that might account for the reduced slope of the response-latency functions in the no-delay condition.

It might be argued that the reduced slope of the response-latency function in the no-delay condition is, in part or whole, a consequence of scaling problems resulting from the longer response latencies. This seems unlikely for several reasons. First, the difference between the longest mean response latency in the no-delay condition and the longest mean latency in the delay condition was only about $50 \mathrm{msec}$. Second, there was no evidence of a decrease in the slope of the response-latency function with increasing response latency in either of the conditions. In the no-delay condition, there was a small positive correlation between the slope of the response-latency function and mean response latency $(r=.39, n=20$, $p>.05$, two-tailed). In the delay condition, there was no evidence of such a correlation $(r=.02)$.

\section{Discussion}

Comparison of the delay and no-delay conditions of Experiment 1 reveals several sizable effects of a preprobe delay. Average response latency in the delay condition, as opposed to the no-delay condition, was reduced by $108 \mathrm{msec}$, with a $68 \%$ reduction in errors. The slope of the combined response-latency function was $22.49 \mathrm{msec} /$ item steeper in the delay condition than in the no-delay condition.

It is somewhat surprising that greater serial-position effects were not found in the no-delay condition of Experiment 1 . Immediately after presentation of the memory set, the trace strength of the last items in the set should be high. In the absence of a preprobe delay, there is little opportunity for the strength of the last items to decay or for the relative strength of the items in the set to be reordered by active rehearsal. Recency effects might therefore be expected. In fact, recency effects are often reported for short delay intervals. On the basis of an examination of studies using varying stimulus presentation rates and preprobe delay intervals, Cavanagh (1976) concluded that recency effects are reliably produced by delays of less than $1 \mathrm{sec}$.

The results of Experiment 1 are similar to those reported by Diener (1988) for memory search tasks not requiring an explicit negative response. The slopes of the combined functions in the delay and no-delay conditions of Experiment 1 are nearly identical to the slopes of the comparable single-response functions from the previous study. However, the absence of a preprobe delay affected positive and negative responses differently. In the no-delay condition, there was a substantial set-size effect for positive responses (18 msec/item), but no effect for negative responses. Although this finding is potentially important to understanding the role of the preprobe delay, a discussion of this issue will follow the presentation of the results of Experiments 2 and 3 .

\section{EXPERIMENT 2}

There are several potential explanations for the reduced set-size effect in the absence of a preprobe delay. It is possible that the time interval is important. The set-size effect might depend on processing that occurs during the delay interval. On the other hand, time itself may not be crucial. The preprobe delay may serve primarily to inform the subject that the memory set is complete and that the test item is forthcoming. If the delay interval serves primarily an informational function, then informing the subject of the set size before a trial begins should restore at least a portion of the set-size effect, even in the absence of a preprobe delay. The task employed in Experiment 2 was similar to the no-delay condition of Experiment 1 in all respects except that the subjects were informed of the size of the memory set before it was presented.

\section{Method}

Subjects. Seven men and 13 women, recruited from undergraduate classes in psychology, participated in Experiment 2. The subjects ranged in age from 17 to 38 years (median age $=20$ years).

Procedure. The procedures employed in Experiment 2 were similar to those employed in the no-delay condition of Experiment 1 , with the following exception. Before each trial, the message "Number of digits in next set: $(n)$ " was displayed on the screen above the instructions to press the space bar for a new trial. This message remained on the screen until the space bar was pressed.

\section{Results}

Response latency and error rates increased with set size in the typical fashion for both positive and negative responses in Experiment 2 (see Figure 2). An ANOVA performed on response latency revealed a significant setsize effect $[F(5,95)=27.95, p<.001]$ and a signifi- 


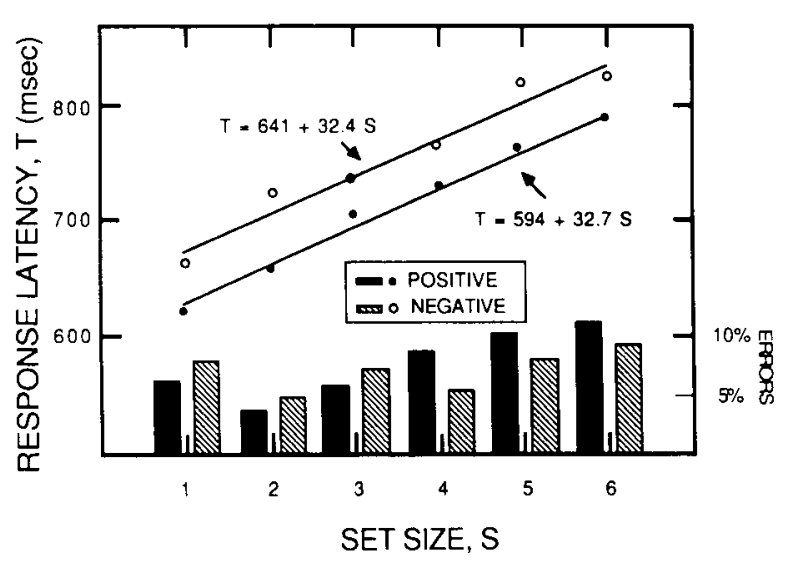

Figure 2. Mean response latency and error rates as a function of set size for positive and negative responses in Experiment 2.

cant response-type effect $[F(1,19)=17.93, p<.001]$ due to the longer latencies of negative responses (i.e., 754 vs. $709 \mathrm{msec}$ ). There was no response type $\times$ set size interaction $[F(5,95)<1]$.

Regression of response latency on set size for positive responses yielded a slope of $32.74 \mathrm{msec} / \mathrm{item}( \pm 6.63$; $r=.98)$ and an intercept of $594 \mathrm{msec}$; for negative responses, the slope was $32.43 \mathrm{msec} /$ item $( \pm 7.38 ; r=$ $.98)$ and the intercept was $641 \mathrm{msec}$. Error rates averaged $7.7 \%$ for positive responses and $6.9 \%$ for negative responses.

The response-latency data from Experiment 2 were compared with the data from the delay condition of Experiment 1 in a condition $\times$ set size $\times$ response type $\times$ subject within-condition ANOVA. There were no significant differences between the two conditions. The main effect of condition was not significant $[F(1,38)=1.55$, $p>.05$ ], nor were the response type $\times$ condition $[F(1,38)<1]$, size $\times$ condition $[F(1,190)=1.25$, $p>.05]$, or response type $\times$ size $\times$ condition $[F(1,38)<1]$ interactions.

Serial-position effects were examined in separate subject $\times$ serial position ANOVAs for each series length. Significant serial-position effects were found for set sizes of two digits $[F(1,19)=14.24, p<.01]$, three digits $[F(2,38)=7.74, p<.01]$, four digits $[F(3,57)=4.85$, $p<.01]$, and five digits $[F(4,76)=5.37, p<.01]$. The linear components of trend analyses were significant for all of these set sizes, indicating recency effects [two digits, $t(19)=-3.81, p<.01$; three digits, $t(38)=-2.81$, $p<.01$; four digits, $t(57)=-2.19, p<.05$; five digits, $t(76)=-2.91, p<.01]$. In addition, there was a significant quadratic (primacy-recency) effect for a set size of five digits $[t(76)=-2.72, p<.01]$.

\section{Discussion}

There were a greater number of significant recency effects in Experiment 2 than in either condition of Experiment 1 . It is not clear why recency effects should be more pronounced as a consequence of informing the subject of the size of the forthcoming set. The latency of positive responses in Experiment 2 was, on average, only about $55 \mathrm{msec}$ lower than in the no-delay condition of Experiment 1 . This difference in response latency seems too small to explain the greater recency effects on the basis of trace-strength decay. It is possible that recency effects in the no-delay condition of Experiment 1 were somehow reduced by the processing necessary to recognize that the test digit was presented and that a response was required.

With the exception of serial-position effects, the results of Experiment 2 are similar to the results of the delay condition of Experiment 1. The slopes of the response-latency functions for combined positive and negative responses in the two experiments differ by less than $2 \mathrm{msec} / \mathrm{item}$, and the error rates are quite similar. Informing the subject of the size of the memory set in the absence of a preprobe delay results in a set-size effect similar to that obtained with a 1-sec delay and no prior information about the set size. Obviously, the time interval itself is not crucial to producing the set-size effect. Rather, it would appear that the preprobe delay functions in a manner similar to information about the size of the memory set-to cause the subject to expect a memory probe at a specific time.

Causing the subject to expect the test digit at a specific point in the series could influence the set-size effect only if this expectancy is relevant to some factor that changes with the size of the memory set. The most obvious candidate for this role is the expectancy of the test digit itself. In the absence of information about the size of the memory set, the subject's expectancy that the test digit is forthcoming might rise as each member of the memory set is presented (see Nickerson, 1967, for a discussion of expectancy effects). A reduction in response latency associated with increased expectancy might offset increased retrieval time for larger sets. In the absence of information about the size of the set, a preprobe delay might control expectancy effects by providing a period during which the subject knows that the next item to be presented will be the test digit.

In memory-search tasks such as those employed in the present study, an increasing expectancy that a response will be required as set size increases is reasonable. Because the tasks are designed so that all set sizes are equally likely, the conditional probability of an immediate response increases as successive digits are presented. Following the first digit, the probability of a response is $1 / 6$. Given that a response is not made to the first digit, the probability that a response will be required immediately following the second digit is $1 / 5$. The probability that a response will be required following the third digit is $1 / 4$ [in general, $1 /(7-n)$, where $n$ is the current set size]. Following the sixth digit, a response is always required. Thus, as successive digits are presented, the objective probability that a response will be immediately required increases.

The nature of many potential explanations of the effect of expectancy on response latency will vary somewhat, depending on the nature of the processes assumed to un- 
derlie performance on the memory-scanning task. With some notable exceptions (e.g., Sternberg's 1966, 1969, original model), most models of performance on the memory-scanning task assume that response latency varies with something like the trace strength of the representation of the test digit in memory (e.g., Baddeley \& Ecob, 1973; Krueger, 1970; Norman \& Wickelgren, 1969). Expectancy cannot sum in a simple way with trace strength. If it did, increasing expectancy would reduce the latency of positive responses and increase the latency of negative responses. Thus, if expectancy increases with set size, the positive-response function would be flatter than would the negative-response function. However, in the no-delay condition of Experiment 1, the slope of the positiveresponse function was steeper than was that of the negative-response function.

It is possible to explain the results of Experiments 1 and 2 in the following way. Assume that items comprising the representation of the positive set in memory vary in trace strength and that the subject can, depending on available cues, treat new items either as additions to the memory set or as the memory probe. As each new item is presented, the subject has access to the trace strength associated with the corresponding memory locus. Items of low trace strength may be added to the memory set or, if perceived as the probe, may elicit a negative response. Items of intermediate strength may also be added to the memory set. If perceived as the probe, these items may require additional processing. Items of high trace strength may elicit an immediate positive response even if not clearly identified as the probe.

In the absence of a preprobe delay or information about set size, uncertainty about whether an item is the memory probe or an addition to the memory set might cause the subject to engage in inefficient processing, perhaps encoding the test digit in memory. The lower the expectancy of the probe, the more likely it is that this inefficient processing will occur. On some proportion of positive trials, however, trace strength will be high enough that the probe will be recognized immediately, even if unexpected. Thus, the effect of expectancy will be somewhat lower for positive items than it will be for negative items.

Other explanations of the present results based on relationships among expectancy, trace strength, and response type are no doubt possible. It is also possible, however, that expectancy effects are independent of the memorysearch process ("additive" in Sternberg's, 1969, parlance). As successive digits are presented and the expectancy of the test digit increases, subjects might be increasingly attentive or simply better prepared to respond. Thus, expectancy effects would merely mask longer search times for larger sets. If the foregoing is correct, it should be possible to observe a decrease in response latency with increasing set size on a task that is similar in stimulus arrangement to the tasks involved in Experiments 1 and 2 but that requires no memory search.

\section{EXPERIMENT 3}

The task employed in Experiment 3 was designed to be similar to the tasks employed in the previous experiments without requiring the subjects to engage in a memory search. The subjects were presented with a series of one to six digits, followed by a visual cue to respond, either immediately (no-delay condition) or after a 1-sec warning interval (delay condition). Upon presentation of the response cue, the subjects were required to indicate whether the final digit in the series was "odd" or "even." Thus, the subjects were required to attend to each digit in the series but only to remember a single digit, regardless of the series length. Changes in response latency as a function of set size on this task should be attributable primarily to changes in expectancy.

If expectancy operates independently of a memory search, it should be possible to observe a decrease in response latency with increasing set size in the no-delay condition. This decrease should be as large as the difference in slope between the no-delay and delay conditions of Experiment 1 (about $-22.5 \mathrm{msec} /$ item). Moreover, the effect should be eliminated or greatly reduced by the introduction of a preprobe delay.

\section{Method}

Subjects. Seven men and 13 women, ranging in age from 17 to 41 years (median age $=18.5$ years), participated in the no-delay condition; 6 men and 14 women, ranging in age from 18 to 41 years (median age $=25.5$ years), participated in the delay condition. The subjects were recruited from a pool similar to that used in the previous experiments

Procedure. The procedures employed in Experiment 3 were similar in most respects to those employed in the previous experiments. On each trial, a series of one to six digits was presented sequentially in the center of the screen at the rate of one digit per second. In the no-delay condition, the response cue was an illuminated square immediately displayed at the same position the digits had appeared. In the delay condition, the response cue followed a 1-sec interval during which the screen was entirely blank. Upon the presentation of the cue, the subjects were required to indicate whether or not the last digit in the series was even. The subjects were instructed to press the $\mathrm{K}$ key on the computer keyboard with the index finger of the right hand for an affirmative response (i.e., to indicate that the last digit was even) and to press the D key with the index finger of the left hand if the last digit was not even. The response cue was displayed until the subject responded.

To avoid confusion, zeros were excluded from the memory set. The set of even digits $(2,4,6,8)$ was therefore smaller than the set of odd digits $(1,3,5,7,9)$. However, the system was programmed so that odd and even digits were equally likely.

Apart from the above-described factors, the procedures followed in Experiment 3 (apparatus, number of trials, error messages, etc.) were identical to those of Experiments 1 and 2.

\section{Results}

Response latency and error rates decreased linearly with series length in the no-delay condition (see Figure 3 ). In the delay condition, there was also a significant decrease in response latency with series length. However, as is evi- 


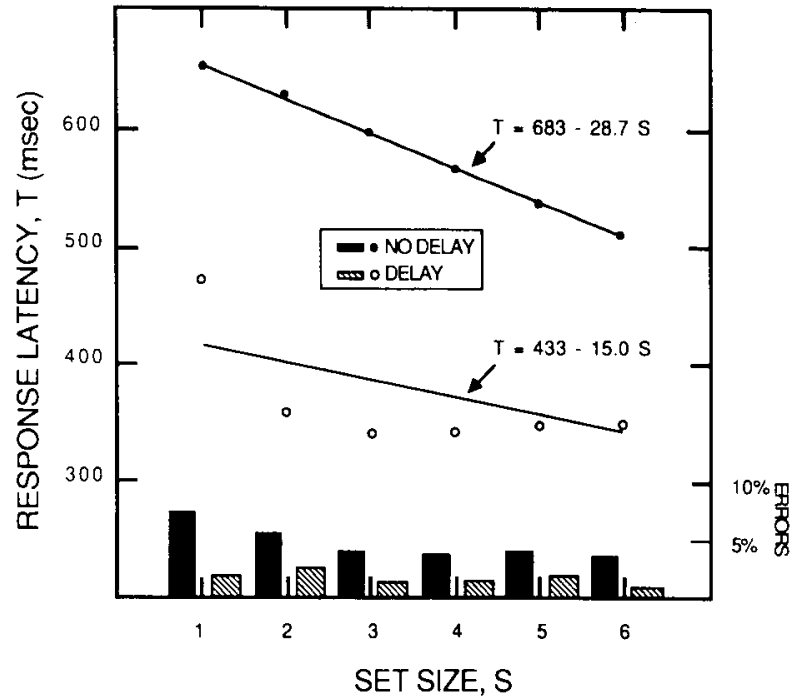

Figure 3. Mean response latency and error rates as a function of set size for the delay and no-delay conditions of Experiment 3.

dent in Figure 3, this decrease was almost entirely attributable to a long response latency in the case of the singledigit series.

A delay $\times$ set size $\times$ response type $\times$ subject withindelay ANOVA confirmed the trends apparent in Figure 3. Mean response latency was significantly longer in the nodelay condition than in the delay condition [i.e., 583 vs. $365 \mathrm{msec} ; F(1,38)=46.76, p<.001]$. The decrease in response latency with set size was significant $[F(5,190)$ $=32.98, p<.001]$. A significant set size $\times$ delay interaction $[F(5,190)=6.69, p<.001]$ confirmed the difference in the slopes between the two conditions. There was no main effect of response type $[F(1,38)<1]$, response type $\times$ delay interaction $[F(1,38)<1]$, nor set size $\times$ response type $\times$ delay interaction $[F(5,190)=$ $1.04, p>.05]$.

Separate subject $\times$ set size $\times$ response type ANOVAs were performed on the response-latency data from the two conditions. Significant set-size effects were found in both conditions [delay condition, $F(5,95)=11.42, p<.001$; no-delay condition, $F(5,95)=34.45, p<.001]$. There were no main effects of response type $[F(1,19)<1$, in both cases] and no response type $\times$ set size interactions $[F(5,95)<1$, in both cases $]$.

In the no-delay condition, the slope of the responselatency function for "even" responses was $-26.09 \mathrm{msec} /$ item $( \pm 4.81 ; r=-.9989)$ and the zero intercept was $674 \mathrm{msec}$; the slope for "odd" responses, the slope was $-31.25 \mathrm{msec} /$ item $( \pm 5.80 ; r=-.9995)$ and the intercept was $692 \mathrm{msec}$. In the delay condition, the slope for "even" responses was $-16.09 \mathrm{msec} / \mathrm{item}( \pm 7.22 ; r=$ -.72; and the intercept was $440 \mathrm{msec})$ and the slope for "odd" responses was $-13.87( \pm 6.16 ; r=-.64)$ and the intercept was $425 \mathrm{msec}$. If the series length of a single digit is deleted from the analysis, the slope for the combined responses in the delay condition was -1.66 $( \pm 5.58 ; r=-.33)$.
In the no-delay condition, error rates averaged $4.8 \%$. In the delay condition, error rates were somewhat lower, averaging $1.5 \%$.

\section{Discussion}

Although the task employed in Experiment 3 differs from the standard memory-search task in a number of ways, the tasks would seem likely to be quite similar with respect to changes in response expectancy with increasing set size. Assuming that the task does not require operations that vary in processing time with changes in set size, the decrease in response latency with increasing set size should be attributable to the effects of expectancy. In the no-delay condition, this effect was about $29 \mathrm{msec} /$ item, more than large enough to account for the reduced set-size effect in the no-delay condition of Experiment 1 . It seems possible to conclude that expectancy operates independently of the memory search.

In the delay condition, response latency for the shortest series was over 100 msec greater than for the longer series lengths. Unlike the delay condition of Experiment 1 , in which the delay interval was marked by an illuminated square, the delay interval in Experiment 3 was designated only by a blank screen, with the illuminated square serving as the cue to respond. Perhaps the subjects failed to recognize the delay interval quickly when it followed a single digit. Following a series of two or more digits, the blank screen might be more salient. There was little variation in response latency for set sizes of two to six digits, indicating that the delay interval served as an effective control for expectancy effects for these series lengths.

Overall response latency in the delay condition was considerably lower than was that in the no-delay condition. Even for the longest series length, there was about a 160 msec advantage in reaction time for the delay condition. This difference suggests that the delay interval does more than control response expectancy. In Experiment 3, both the identity of the test digit and the decision required ("Is the digit even?') were known to the subject during the delay interval. Thus, the delay interval could be used to prepare to execute a response. In the typical memoryscanning task, the identity of the test digit is not known during the delay interval and the subject cannot prepare fully for a response.

\section{GENERAL DISCUSSION}

Taken together, the results of the present experiments indicate that, in the absence of effective controls, the memory-scanning task is subject to expectancy effects that increase with set size in such a way as to reduce the setsize effect. The increasing expectancy of an immediate response might improve the subject's performance in several ways. First, as successive digits are presented, the subject might be increasingly attentive to the presence of the response cue, reducing the latency of recognizing that a response is required. Second, the subject might pre- 
pare to execute a response by, for example, rehearsing contingent response strategies (e.g., "if a 2, press $\mathrm{K}$, otherwise press D"). Either of these strategies could reduce response latency as response expectancy increases with set size, thereby reducing the manifest set-size effect.

The primary effect of a preprobe delay is to control expectancy effects across sets of different sizes by providing a period during which it is known that a response will be required. It seems reasonable to ask whether a delay interval of the magnitude employed in the present study is sufficient to eliminate expectancy effects or whether there are residual expectancy effects despite the preprobe delay.

Several sources of evidence indicate that the magnitude of the set-size effect is estimated accurately using delay intervals of about $1 \mathrm{sec}$. First, the magnitude of the setsize effect in Experiment 2 of the present study, in which the subjects knew the size of the set before it was presented, was nearly equal to that of the delay condition of Experiment 1. Second, the fixed-set procedure, in which the size and the content of the positive set are kept constant across blocks of trials, produces slopes of about the same magnitude as those produced by the varied-set procedure (e.g., Sternberg, 1966, 1969). Third, variation in the duration of the delay interval beyond a rather brief period appears to have little effect on the magnitude of the set-size effect.

Clifton and Birenbaum (1970) presented subjects with one to seven digits at a rate of $1.5 \mathrm{sec} /$ digit. Three different preprobe delay intervals were used: $0.8,2.8$, and $4.8 \mathrm{sec}$. There was no significant set size $\times$ delay interaction for either positive or negative responses. Aubé and Murdock (1974) presented one to eight digits at the rate of $250 \mathrm{msec} /$ digit, followed by a preprobe delay of $300 \mathrm{msec}$. The slopes of the response-latency functions in this study were considerably smaller than values typically reported for longer delay intervals $-23 \mathrm{msec} / \mathrm{item}$ for positive responses and $16 \mathrm{msec} /$ item for negative responses (computed from the published data by Cavanagh, 1976). Thus, a delay interval somewhere between 300 and $800 \mathrm{msec}$ long appears to be sufficient for a setsize effect of the usual magnitude.

Although the magnitude of the set-size effect does not seem to be affected significantly by the duration of the preprobe delay beyond some minimum value, the difference in the slopes of positive and negative response functions may be influenced by the preprobe delay. Cavanagh (1976) concluded from a survey of memory-scanning studies that the slopes of response-latency functions for positive responses are usually slightly steeper than those for negative responses, although this difference is seldom statistically significant. The difference in the slopes of positive- and negative-response functions increases, often attaining statistical significance, as the rate of presentation of the members of the positive set increases and the preprobe delay decreases (these factors are usually confounded). Cavanagh attributed this increase to the absence of the opportunity for rehearsal when members of the positive set are presented rapidly.
The results of the present study fit the pattern observed by Cavanagh but suggest a different interpretation. In the delay condition of Experiment 1, the slope of the positiveresponse function was somewhat steeper than that of the negative-response function. In the no-delay condition, the negative-response function was flat and significantly different from the 18-msec/item slope of the positive function. However, in Experiment 2, in which the subjects were informed of the size of the forthcoming memory set, the slopes of the positive- and negative-response functions were equal. This pattern of results suggests that uncertainty about when a response will be required has a greater effect on negative trials than it does on positive trials.

The greater effect of expectancy on negative trials might be due to the information available to prompt a response. On negative trials in the no-delay condition of Experiment 1 , the only cue that a response was required was the display format of the test digit. On positive trials, the identity of the test digit could also serve as a cue to respond. If a subject, not expecting the memory probe, failed to attend to the display format but instead attended to the identity of the test digit, a response might have been prompted by the repetition of the digit on positive trials. On negative trials, the subject was required to shift attention from the identity of the test digit to its display format in order to know that a response was required.

As more digits were presented and the expectancy of a test digit increased, attention might have been increasingly deployed toward the display format. This deployment of attention would cause a decrease in response latency on negative trials so as to reduce the set-size effect. On positive trials, however, the matter is more complex. Attention to the display format would shift attention from the identity of the test digit. The subject might first attend to the display format and then to the digit's identity, reducing processing efficiency somewhat and attenuating expectancy effects on positive trials.

Previous research has shown that subjects can easily base responses solely on the identity of the test digit. Diener (1988, Experiment 1) used a task in which subjects were presented with a series of three to eight digits, the last member of which had occurred previously at some point in the series. The subjects were instructed to press a single key as soon as any digit was repeated. Under these conditions, subjects could know that a response was required only after recognizing that the test digit had been previously displayed. Response latencies on this task were somewhat lower than were those obtained in the present study, averaging $566 \mathrm{msec}$ and decreasing slightly with set size at the rate of $6.24 \mathrm{msec} / \mathrm{item}$.

Similar processes might account for Cavanagh's (1976) observation that brief delay intervals and rapid presentation rates increase the difference in the slopes of positiveand negative-response functions. However, because in most studies the test digit is identified only by the preprobe delay, the difference in slopes cannot be based on the consequences of attending to an extrinsic cue. Following a brief delay interval, the subject might be uncertain that a response is required, either because the interval is 
difficult to detect or because it takes longer than the delay interval for the response requirement to register fully. This uncertainty would increase response latency disproportionately for smaller set sizes where the test digit is unexpected. The effect would be lessened on positive trials because the identity of the test digit can serve as a response cue.

\section{REFERENCES}

AuBÉ, M., \& Murdock, B. (1974). Sensory stores and high-speed scanning. Memory \& Cognition, 2, 27-33.

BadDEley, A. D., \& Eсов, J. R. (1973). Reaction time and short-term memory: Implications of repetition effects for the high-speed exhaustive scan hypothesis. Quarterly Journal of Experimental Psychology, 25, 229-240.

Cavanagh, J. P. (1976). Holographic and trace strength models of rehearsal effects in the item recognition task. Memory \& Cognition, 4, 186-199.

Clifton, C., \& Birenbaum, S. (1970). Effects of serial position and delay of probe in a memory scan task. Journal of Experimental Psychology, 86, 69-76.

DIENER, D. (1988). Absence of the set-size effect in memory-search tasks in the absence of a preprobe delay. Memory \& Cognition, 16, 367-376.

Egeth, H., Marcus, N., Bevan, W. (1972). Target-set and responseset interaction: Implications for models of human information processing. Science, 176, 1147-1148.

KRUEGer, L. E. (1970). Effect of stimulus probability on two-choice reaction time. Journal of Experimental Psychology, 84, 377-379.

Nickerson, R. S. (1967). Expectancy, waiting time and the psychological refractory period. Acta Psychologica, 27, 23-34.

Norman, D. A., \& WiCkelgren, W. A. (1969). Strength theory of decision rules and latency in retrieval from short-term memory. Journal of Mathematical Psychology, 6, 192-208.

STERNBERG, S. (1966). High-speed scanning in human memory. Science, $153,652-654$.

SteRnBerG, S. (1969). Memory scanning: Mental processes revealed by reaction-time experiments. American Scientist, 57, 421-457.

(Manuscript received August 1989; revision accepted for publication January 31, 1990.) 